\title{
THE RELATION OF UPPER RESPIRATORY INFECTIONS TO RHEUMATIC FEVER IN CHILDREN
}

\section{Antihemolysin Titres in Respiratory Infections and Their Significance in Rheumatic Fever in Children ${ }^{1}$}

\author{
By MAY G. WILSON, GEORGE W. WHEELER, AND \\ MARGUERITE M. LEASK \\ (From the New York Hospital and the Department of Pediatrics, \\ Cornell University Medical College, New York)
}

(Received for publication December 1, 1934)

In the preceding article (1) evidence was presented which would not support the conception of a specific etiological relationship between respiratory infections and rheumatic fever in children. The designation, "streptococcal" respiratory infection, based solely on bacteriological findings, was questioned.

During these observations as to the relation between respiratory infections and rheumatic fever, an opportunity presented itself for obtaining information as to the immunological response of children experiencing respiratory infection and rheumatic recurrences. The serological method described by Todd (2) in 1932 for titrating antistreptolysin in the blood serum, by which a diagnosis of preceding hemolytic streptococcal infection may be made, was utilized.

From limited observations of normal individuals, mainly adults and others suffering from miscellaneous and streptococcal infections, Todd (3) and Coburn and Pauli (4) considered the normal range of antistreptolysin in serum to be from 5 to 100 units, and values of 200 or more as significant of preceding streptococcal infections. They also observed the antistreptolysin content to be consistently high during rheumatic fever and considered this as immunological evidence that the hemolytic streptococcus initiates the rheumatic process.

Myers and Keefer (5), however, obtained an average in normals of 200 units, with a somewhat higher average, 500 units, in rheumatic fever, but they did not attribute etiological significance to this finding.

Wilson, Wheeler and Leask (6), in a preliminary report on the antistreptolysin content of the

1 This study was conducted under a special grant from the Commonwealth Fund. serum in children, found that this antibody apparently passes through the placenta, being present in new-born infants. An analysis of the average titres for various age groups indicated a definite age correlation, the lowest values being obtained in the age period between three months and three years. It is of interest that the values obtained from sixteen mothers was within the normal range reported by Todd (3), but was significantly lower than those obtained for children six to sixteen years of age by these observers. The possible significance of this difference will be considered later.

Early in our studies it was apparent that single determinations of antistreptolysin were of little value because of the wide normal range and frequent presence of elevated titres for prolonged periods of a year or more. It was, therefore, considered advisable to obtain serial specimens at regular frequent intervals during the entire period of the observations. By this procedure it was possible to correlate the clinical course with the bacteriological and immunological findings of a representative series of subjects.

\section{SUBJECTS AND METHOD}

Todd's method for determining antistreptolysin in the blood serum was followed. The test is based on the presence in the blood serum of an agent which is capable of combining with, and neutralizing, the hemolysin in filtrates of broth cultures of hemolytic streptococcus. Todd (2) prescribed the following standard units:

Red blood cells (rabbit) -0.5 cc. of a 5 per cent suspension of washed cells

Antigen-Streptolysin-Minimum hemolytic dose (M. H. D.). Smallest quantity of streptolysin filtrate that will completely hemolyze one unit of rabbit cells in one hour at $37^{\circ} \mathrm{C}$. This is determined by titration and is expressed in cc. 
Antibody-Antistreptolysin-Neutralizing dose (N. D.). Smallest quantity of patient's serum, inactivated 15 minutes at $55^{\circ} \mathrm{C}$., that will combine with and completely neutralize 2.5 units of streptolysin in 15 minutes at $37^{\circ} \mathrm{C}$. This is determined by titration and is expressed in ordinary or decimal fractions of a cc. or in Todd units (3), which are the reciprocals of the decimal fractions and indicate the number of neutralizing doses in $1 \mathrm{cc}$. of serum.

The methods for the preparation of these reagents are given in detail in the appendix.

By adding diminishing quantities of serum to tubes containing 2.5 units of streptolysin, allowing 15 minutes at $37^{\circ} \mathrm{C}$. for neutralization to take place, then adding one unit of red blood cells to each tube and incubating for one hour, the smallest amount of serum that would completely inhibit hemolysis was determined. This was recorded as the titre of the serum.

Adequate data for analysis was obtained for eighty rheumatic subjects who were under close observation for a period of from twelve to eighteen months. From serial antistreptolysin determinations, the basal level was obtained for each child. The lowest titre during the period of observation, which was maintained in apparent health, was selected as the basal level. In the majority of instances, periods of two to six months free from symptoms of infection preceded this value. The rise of titre above the basal level or above the level maintained during apparent health before the occurrence of respiratory infection or rheumatic activity was calculated.

A respiratory infection was considered a "streptococcal" infection if hemolytic streptococci were present in the pharyngeal flora during the respiratory infection, in contra-distinction to respiratory infections unassociated with hemolytic streptococci in the flora of the throat. Respiratory infections were graded as mild or severe based on local and constitutional symptoms.

The following were considered as rheumatic recrudescences: joint pains, purpura, arthritis, nodules, chorea and carditis.

\section{OBSERVATIONS}

In view of the observed frequency of respiratory infection and the high seasonal carrier rate of hemolytic streptococci in the pharyngeal flora of these children, it was obviously impossible to obtain antistreptolysin determinations in children free from either during the entire period of the observations. In this study the basal values obtained for 80 rheumatic subjects during apparent health showed a range of from 25 to 715 units with an average of 135 units. This is somewhat lower than that observed in our preliminary study (6). The opportunity for special selection of subjects and the longer period of observation probably accounts for this difference. The antistreptolysin titre remained remarkably constant at various levels during periods when the subjects were free from respiratory infection. It is possible that the high basal levels which were maintained by apparently healthy children may represent chronic unrecognized infection or some infection occurring previous to our observation, as it was obviously impossible to eliminate subjects who had had respiratory infection the previous year. It would seem reasonable to consider these observations on the antistreptolysin content of the serum as representative for inactive rheumatic children, six to sixteen years old, in apparent health, although possibly not comparable to normal values given for adults.

A comparison of the range of antistreptolysin titre, the average titre and the rise observed during apparent health and infection is of interest. There was no significant difference in the range of antistreptolysin titre observed in subjects $d u r$ ing health and during respiratory and rheumatic infections. There was no appreciable difference in the average titre of antistreptolysin obtained for subjects during respiratory infections not associated with hemolytic streptococci and similar infections associated with hemolytic streptococci in the pharyngeal flora.

Following respiratory infections irrespective of the bacteriological findings, a rise in titre was consistently although not invariably observed. The average rise and the percentage showing rise in titre was slightly higher following respiratory infections not associated with hemolytic streptococci in the pharyngeal flora, than following similar infections thus associated. The contrary might have been expected. Greater increments and higher percentage of subjects exhibiting a rise in titre were observed following severe respiratory and "streptococcal" infections than mild ones. The degree of rise was not always directly related to the severity of the respiratory infection or to the bacteriological findings. There was consider- 
TABLE I

Antistreptolysin titres in respiratory infections, their significance in rheumatic fever in children

\begin{tabular}{|c|c|c|c|c|c|c|c|c|c|c|c|c|c|}
\hline & & \multirow{3}{*}{$\begin{array}{c}\begin{array}{c}\text { Basal } \\
\text { values }\end{array} \\
\mathrm{I}^{*}\end{array}$} & \multicolumn{4}{|c|}{ Respiratory infection } & \multicolumn{4}{|c|}{$\begin{array}{c}\text { "Streptococcal" respiratory } \\
\text { infection }\end{array}$} & \multicolumn{3}{|c|}{$\begin{array}{l}\text { Not associated with } \\
\text { respiratory infection }\end{array}$} \\
\hline & & & \multicolumn{2}{|c|}{ Mild } & \multicolumn{2}{|c|}{ Severe } & \multicolumn{2}{|c|}{ Mild } & \multicolumn{2}{|c|}{ Severe } & \multicolumn{2}{|c|}{$\begin{array}{c}\text { Hemolytic } \\
\text { streptococcus } \\
\text { in } \\
\text { throat } \\
\text { culture }\end{array}$} & \multirow{2}{*}{$\begin{array}{c}\begin{array}{c}\text { Nega- } \\
\text { tive } \\
\text { throat } \\
\text { cul- } \\
\text { ture }\end{array} \\
\text { A }\end{array}$} \\
\hline & & & $\mathbf{I}$ & $\mathrm{A}^{*}$ & $\mathbf{I}$ & $\mathbf{A}$ & $\mathbf{I}$ & $\mathbf{A}$ & $\mathbf{I}$ & $\mathbf{A}$ & $\mathbf{I}$ & $\mathbf{A}$ & \\
\hline \multicolumn{2}{|l|}{ Number of cases } & 80 & 30 & 17 & 10 & 6 & 45 & 22 & 57 & 20 & 39 & 11 & 11 \\
\hline Antistreptolysin & Range & $\begin{array}{r}25 \\
715\end{array}$ & $\begin{array}{r}71 \\
830\end{array}$ & $\begin{array}{r}25 \\
250\end{array}$ & $\begin{array}{r}71 \\
500\end{array}$ & $\begin{array}{r}200 \\
1000\end{array}$ & $\begin{array}{r}33 \\
830\end{array}$ & $\begin{array}{r}50 \\
333\end{array}$ & $\begin{array}{r}50 \\
620\end{array}$ & $\begin{array}{r}25 \\
1600\end{array}$ & $\begin{array}{r}50 \\
333\end{array}$ & $\begin{array}{r}62 \\
830\end{array}$ & $\begin{array}{r}55 \\
333\end{array}$ \\
\hline Units per cc. & Average & 135 & 244 & 130 & 254 & 366 & 171 & 175 & 198 & 415 & 165 & 262 & 163 \\
\hline \multirow{2}{*}{$\begin{array}{l}\text { Cases with rise above } \\
\text { basal level }\end{array}$} & Number & & 19 & 8 & 7 & 4 & 19 & 7 & 29 & 16 & 19 & 4 & 4 \\
\hline & Per cent & & 63 & 47 & 70 & 67 & 42 & 32 & 51 & 80 & 49 & 36 & 36 \\
\hline \multirow[t]{2}{*}{ Rise in units per cc. } & Range & & $\begin{array}{r}0 \\
300\end{array}$ & $\begin{array}{r}0 \\
130\end{array}$ & $\begin{array}{r}0 \\
360\end{array}$ & 900 & $\begin{array}{r}0 \\
133\end{array}$ & $\begin{array}{r}0 \\
100\end{array}$ & $\begin{array}{r}0 \\
300\end{array}$ & $\begin{array}{r}0 \\
1250\end{array}$ & $\begin{array}{r}0 \\
200\end{array}$ & $\begin{array}{r}0 \\
200\end{array}$ & $\begin{array}{r}0 \\
200\end{array}$ \\
\hline & Average & & 85 & 55 & 152 & 210 & 45 & 49 & 97 & 212 & 58 & 140 & 65 \\
\hline
\end{tabular}

* I = inactive rheumatic.

$\mathbf{A}=$ active rheumatic.

able individual variation in the degree of serological response to what appeared to be a common source of hemolytic streptococcal infection.

It is of passing interest that about 50 per cent of the subjects carrying hemolytic streptococci in the throat during apparent health exhibited a slight rise in antistreptolysin titre.

The low average antistreptolysin titres observed by us (in preliminary studies) in average normal infants and children under the age of five years and by Todd (3), Coburn and Pauli (4) in adults may be related to the relative infrequency of respiratory infections at these age levels compared with the observed high incidence of respiratory infections in children five to sixteen years of age.

Following respiratory infections, the antistreptolysin curve was characterized by a steplike elevation within one to three weeks of the onset, rising to a peak and falling either by lysis within one to two months or remaining plateaulike at intermediate levels for longer periods, showing successive peaks following repeated respiratory infections.

The findings in rheumatic subjects during inactive periods and during respiratory infection must be considered in evaluating the significance of the antistreptolysin content of the serum during rheumatic activity.

Two-thirds of the subjects experiencing rheumatic recrudescences, unassociated with respiratory infections, did not show a rise of antistreptolysin titre. The average values obtained during rheumatic activity associated with respiratory infection and "streptococcal" respiratory infection were significantly elevated, but are comparable to values obtained in inactive rheumatic subjects during similar infections. The slightly higher average values, which obtained in the group of active rheumatic subjects who experienced severe "streptococcal" respiratory infection, were influenced by the occurrence in five subjects of extremely high antistreptolysin values during intercurrent severe streptococcal infection (see protocol of Case 4, C. V.).

The rise and level of antistreptolysin in serum following respiratory infections seemed closely related to the extent of the constitutional and local symptoms associated with the respiratory infection, irrespective of the presence of hemolytic streptococci in the flora of the throat.

The antistreptolysin curves observed in subjects who developed respiratory infections either simul- 
taneously with, or during, rheumatic activity were similar to those described in subjects experiencing respiratory infections alone and bore no relation to the clinical course of rheumatic activity.

Antistreptolysin curves observed during rheumatic activity either maintained the same level noted before the onset of rheumatic activity or assumed the trend of curves observed during respiratory infections. A sharp rise of titre at the onset of rheumatic activity, unaccompanied by respiratory infection, which has been described by Coburn and Pauli, was not obtained. High titres during rheumatic activity were observed in subjects who experienced simultaneously rheumatic activity and respiratory infection. Other subjects maintained a high antistreptolysin level during rheumatic activity which had been present previously for a period of months. A rise of titre was frequently noted during the course of rheumatic activity, following intercurrent respiratory infection, indicating the ability of the subject to react to streptococcal infection. matic fever may be more clearly seen in the following protocols of representative cases.

\section{PROTOCOLS}

Case 1, E. Mc. Girl, six years of age, suffered from first attack of rheumatic infection (chorea) March 1933, from which she made an uneventful recovery in June. During the year of observation 1933-1934 she did not exhibit any evidence of rheumatic activity, although experiencing nine respiratory infections associated with the predominance of hemolytic streptococci in the flora of the throat on four occasions. She also exhibited, in December, a significant rise of antistreptolysin in the serum to 500 units, following a respiratory infection. This case illustrates the presence of "hemolytic streptococcal" infection in a susceptible rheumatic subject, unaccompanied by rheumatic activity.

Case 2, V. B. Girl, six years of age, had her first attack of rheumatic infection (arthritis) in May 1933, followed by an attack of chorea in June, lasting six weeks. In July, 1933, tonsillectomy was performed, and the child remained free from rheumatic activity until June 2,1934, at which time she had a second attack of chorea. In October and November, 1933, she experienced two severe respiratory infections (unassociated with

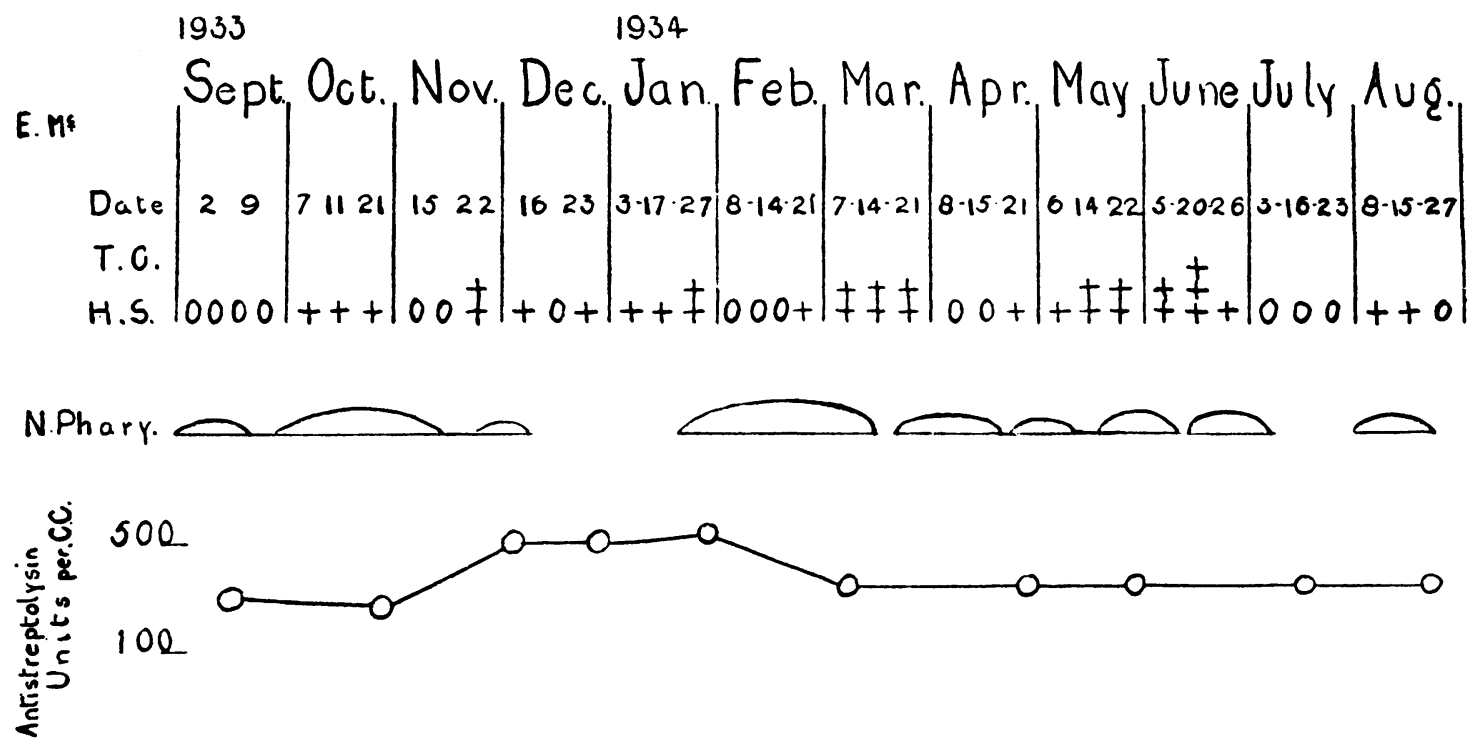

Chart 1. Data on Case 1, E. Mc.

There did not appear to be any relation between the type of rheumatic activity and the antistreptolysin level. Low and high titres were observed in subjects during the last weeks of a fatal rheumatic carditis.

The relation between antistreptolysin content in the serum during respiratory infections and rheu- hemolytic streptococci in the pharyngeal flora) which were followed by a significant rise of antistreptolysin titre, 615 units; and in February and March, 1934, three respiratory infections (with hemolytic streptococci in the throat) which were followed by a comparable increase in antistreptolysin. Rheumatic fever did not follow any of the respiratory infections. The chorea which occurred in June 1934 was preceded by two 
19331934

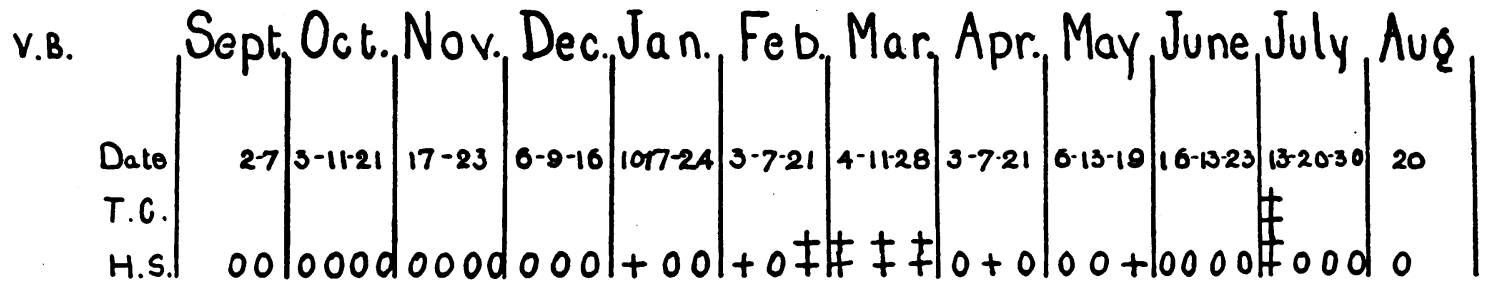

Resp.Inf.
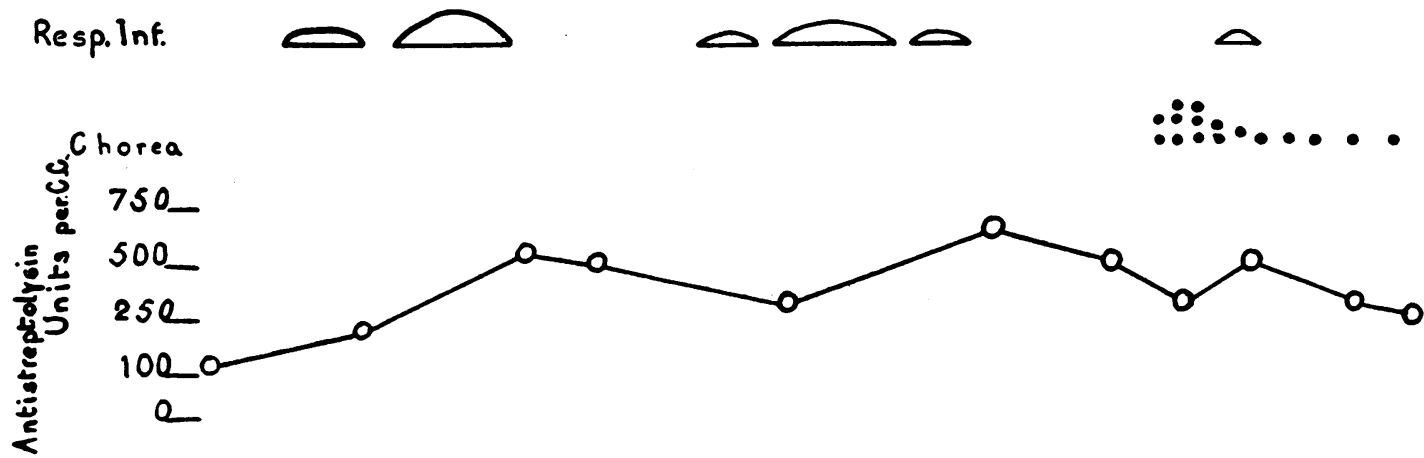

Chart 2. Data on Case 2, V. B.

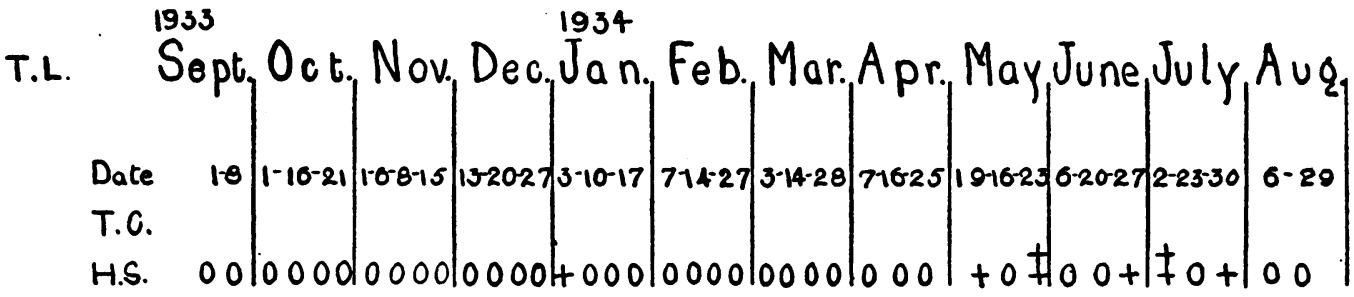

N.Phary.-
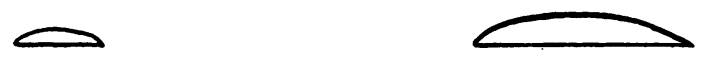

Sinusitıs -

Chorea-
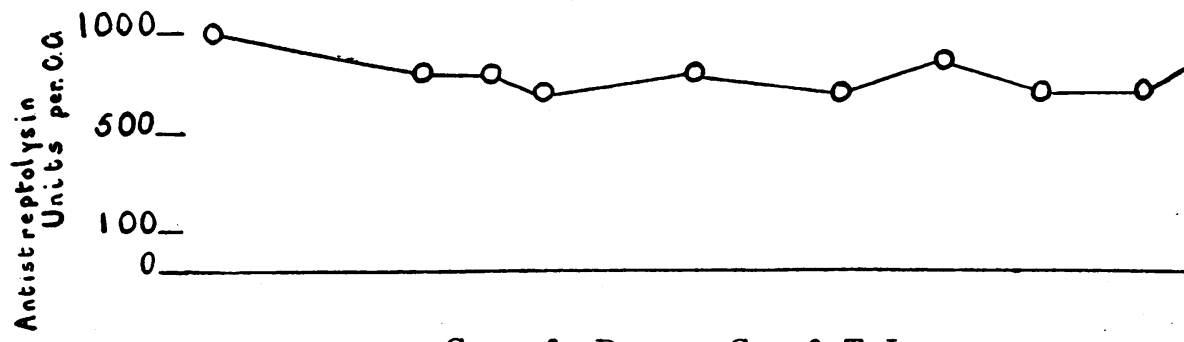

Chart 3. Data on Case 3, T. L. 
months of good health and was not accompanied by a significant rise in antistreptolysin titre. This case illustrates the rise of antistreptolysin in serum following a respiratory infection (non-streptococcal).

Case 3, T. L. Boy, ten years of age, had his onset of rheumatic disease (severe chorea) in September, 1932, from which he recovered in November, 1932. He had recurrent mild attacks of chorea in the summers of 1933 and 1934, of three weeks duration. In September, 1933, he was well and free from respiratory or rheumatic activity. At this time determination of the antistreptolysin content of the serum revealed a titre of a recurrence (rheumatic arthritis and carditis). He remained at Convalescent Cottages from March 31, 1933, until September, 1933, when he returned to the clinic.

In the fall of 1933 hemolytic streptococci were absent from the pharyngeal fiora. He suffered repeated respiratory infections coincident with joint pains. In the first week of December there occurred simultaneous symptoms of a respiratory infection and rheumatic fever characterized by joint pains, arthritis and carditis. He was admitted to the pavilion of the New York Hospital and while there, on February 18, developed scarlet fever during the height of a severe carditis accompanied by con-
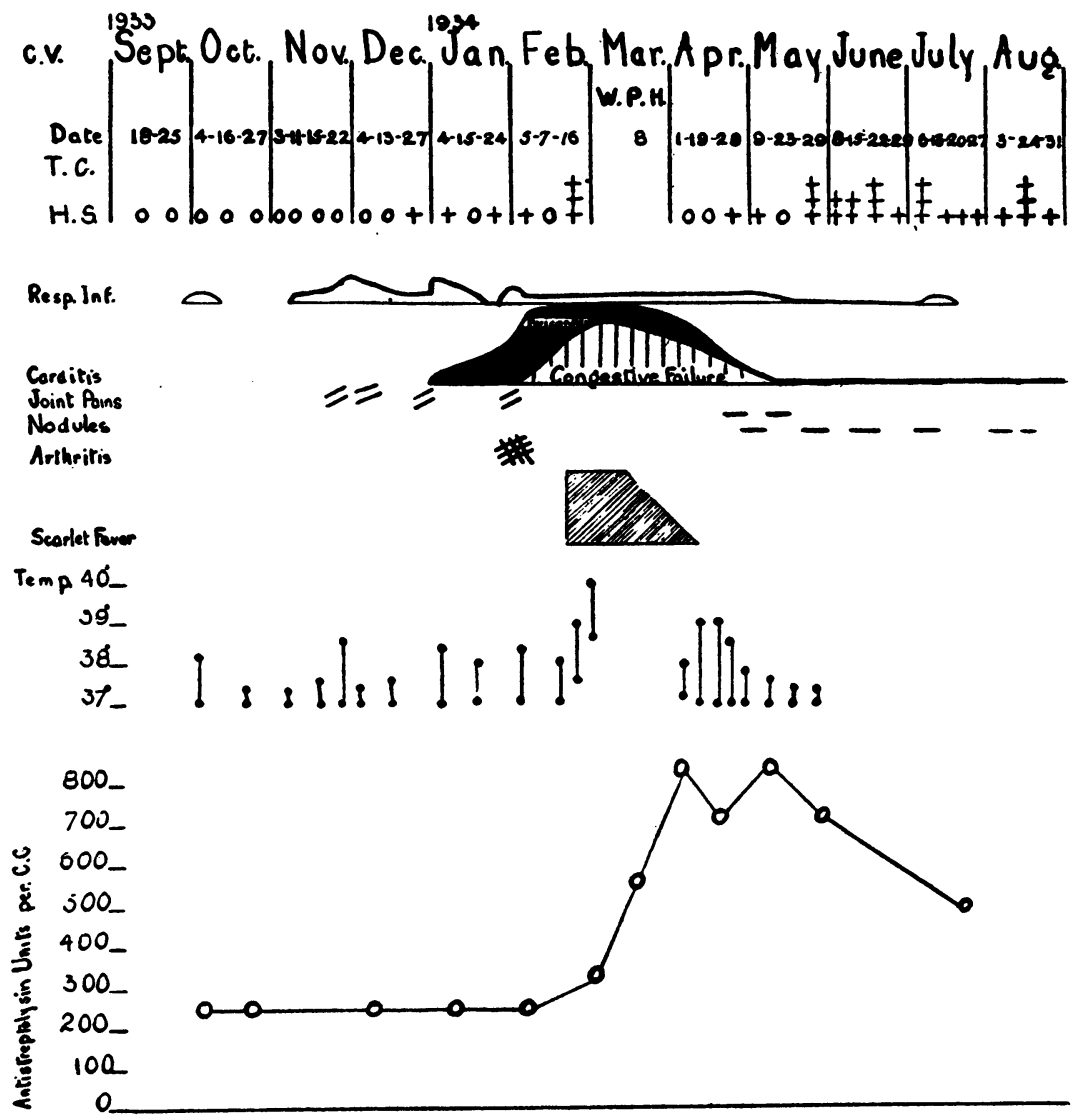

Chart 4. Data on Case 4, C. V.

1,000 units; repeated examinations during the year gave values between 830 and 1,000 units. Hemolytic streptococci were not recovered from the pharyngeal flora until May, 1934. The occurrence of a sinusitis, following a respiratory infection, did not appear to influence the antistreptolysin titre. This case illustrates the persistent presence of high titre for a period of one year in a child during health, respiratory infection and rheumatic activity.

Case 4, C. V. Boy, six and a half years of age, had his onset of rheumatic activity (arthritis and chorea) at the age of three years. In March, 1933, he suffered gestive failure. He was removed to Willard Parker Hospital for a six week period and returned to the New York Hospital, April 6. At that time there was still evidence of congestive cardiac failure and nodules.

Of particular interest is the antistreptolysin curve during this period. It can be seen in the accompanying graph, Chart 4, that the onset of rheumatic fever in December was not accompanied by a rise in antistreptolysin titre, while the occurrence of scarlet fever in February was followed by a significant rise in titre.

Case 5, J. V. Boy of fifteen years, who has been under our observation since he was eight years old, had 
his onset of rheumatic infection at the age of five years and has suffered repeated attacks of arthritis, carditis and nodules. He has been free from rheumatic activity since the age of thirteen.

Hemolytic streptococci were recovered frequently in the flora of the throat from October, 1933, to July, 1934. On February 16, 1934, he suddenly complained of abdominal pain, tachycardia and fever. On examination he showed a red throat, roentgenologic evidence of ethmoiditis and sinusitis, and electrocardiographic examination revealed a partial heart block of but a few days duration. After a symptomless period of two weeks he was sent home and there suffered from further symptoms characterized by fever, arthritis and nodules. nosis). On July 28, following exposure to chilling (while wading the previous day), he suffered from simultaneous onset of symptoms of respiratory infection and rheumatic arthritis and was admitted to New York Hospital pavilion, August 9. During his hospital period of four months, he exhibited three rheumatic recrudescences, characterized by intercurrent symptomless periods of ten days.

In November, he returned home and was well for six weeks. On December 9, he reported to clinic for examination and was found to be free from respiratory or rheumatic infection. On his way home from clinic he was caught in a snowstorm and arrived home cold and wet. That night he complained of fever and joint

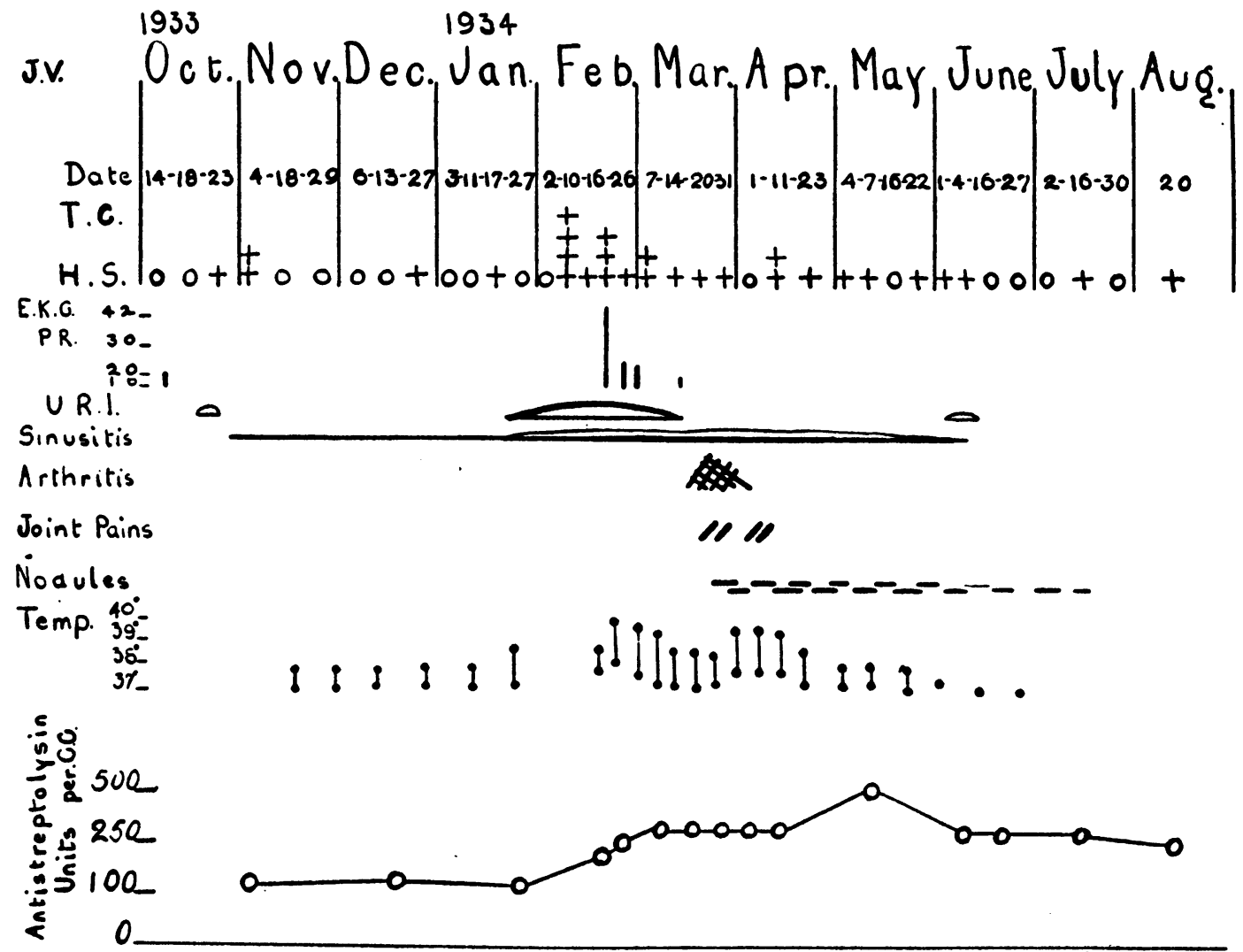

Chart 5. Data on Case 5, J. V.

The antistreptolysin curve is of interest because it shows a step-like elevation of titre, which would appear to be related to the presence of the respiratory infection rather than the clinical course of the rheumatic infection that was simultaneously present. The highest titre was observed during convalescence.

Case 6, A. A. A boy of eight years had his onset of rheumatic fever (arthritis) at the age of five years and repeated attacks at six and seven years, associated with rheumatic carditis. In July, 1933, he was free from rheumatic symptoms and was found to have chronic cardiac valvular disease (mitral insufficiency and ste- pains. On examination he was found to have a temperature of $38^{\circ} \mathrm{C}$, red throat, stuffy nose. During the night he developed an arthritis and symptoms of carditis, associated in the following weeks with nodules. The antistreptolysin curve during this period is of interest in that it showed during these two different episodes no rise in titre at the onset or during rheumatic exacerbations.

This case also illustrates the frequency with which exposure and chilling are followed by simultaneous onset of respiratory infection and rheumatic activity. 


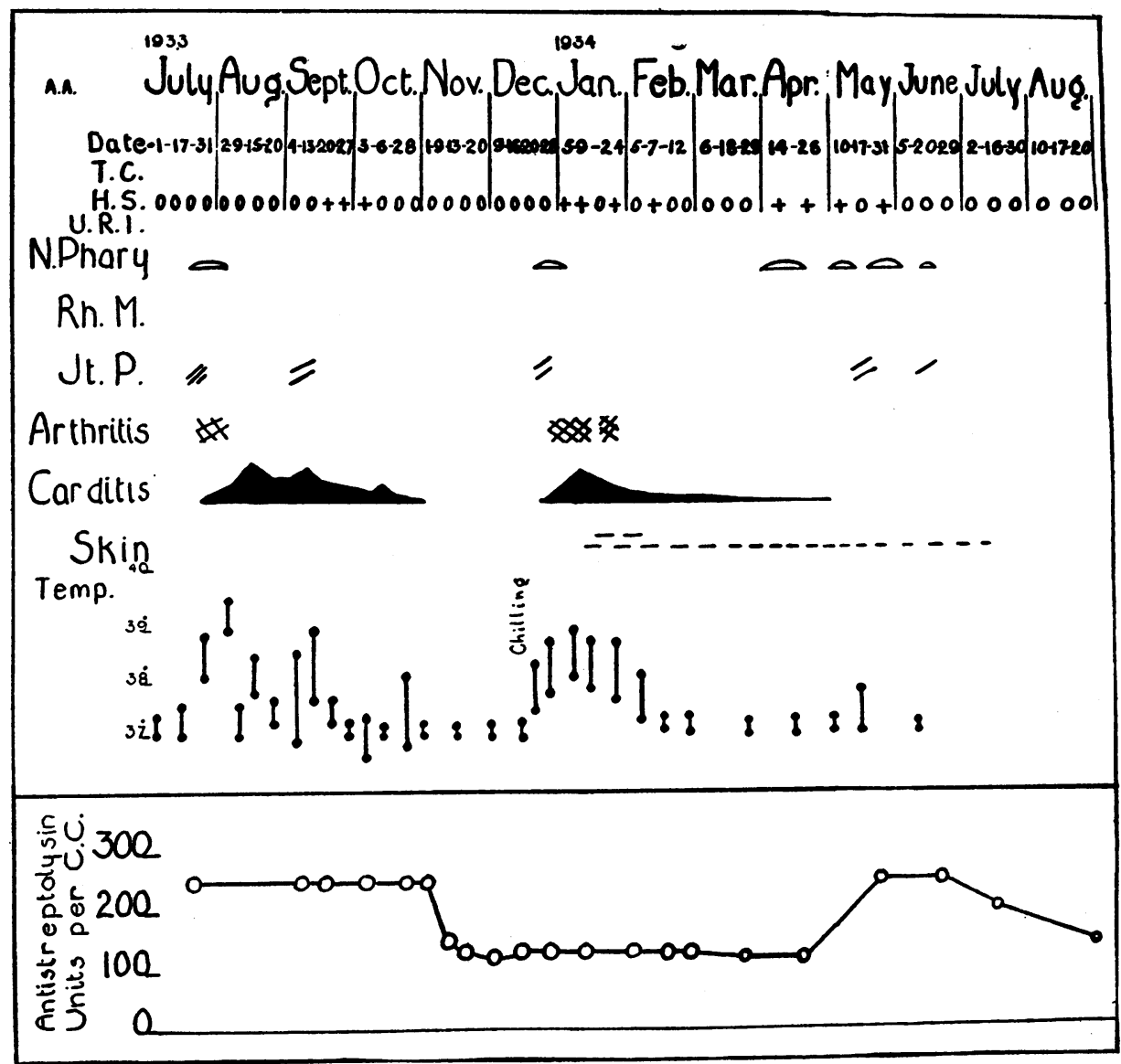

Chart 6. Data on Case 6, A. A.

\section{COMMENT}

At the outset, it was arbitrarily assumed first, that respiratory infections associated with the presence of hemolytic streptococci in the flora of the throat were to be designated as "streptococcal " infections, and second, that a rise in the antistreptolysin titre of the serum was immunological evidence of a previous streptococcal infection. These criteria, we believe, are in accord with the statements of other observers in similar investigations.

The antistreptolysin content of the serum following such infections as scarlet fever, erysipelas and severe streptococcal respiratory infections associated with local and constitutional symptoms has been found to be significantly increased by all observers.

Of particular interest is the rise in titre in twothirds of subjects following upper respiratory infections unassociated with hemolytic streptococci in the pharyngeal flora. One must assume either that hemolytic streptococci were present although not found on examination of repeated throat cultures, or that the antistreptolysin content of the serum is not strictly type specific as Todd (3) believes, and that possibly partially hemolytic streptococci (alpha prime) predominant in the pharyngeal flora may play a rôle. Again, a rise in titre may be due to non-specific stimulation of this antibody during infection.

Irrespective of the significance of these findings, it is evident from our data that the antistreptolysin content of the serum during rheumatic activity appears to be related to the associated respiratory infection rather than to the rheumatic process. This view would seem to be supported by the low titres observed (in the necessarily limited number of observations) in subjects experiencing rheumatic activity unassociated with respiratory infection. It is also worthy of 
note that two-thirds of the total respiratory infections experienced by these 80 subjects were unassociated with rheumatic activity. Of the remaining third, two-thirds occurred either simultaneously with, or during, the rheumatic episode.

The wide normal range of antistreptolysin titre, the individual variation in response to streptococcal infection, the frequency of respiratory infections observed in these subjects, as well as the high carrier rate of hemolytic streptococci, make it difficult to evaluate their possible significance and relation to rheumatic fever. However, it would appear to be reasonable to state that our data do not indicate that the presence of hemolytic streptococci in the pharyngeal flora or that the presence in high titre of antistreptolysin in the serum is necessarily conclusive evidence of a streptococcal respiratory infection.

However, if we accept the criteria mentioned as bacteriological and immunological evidence of "streptococcal " respiratory infection, it is apparent from our observations that "streptococcal" respiratory infection did not, in the majority of cases, activate the rheumatic process and that a rise of antistreptolysin was not a constant accompaniment of rheumatic activity. Its presence appeared to be more frequently related to the coincidental occurrence of a respiratory infection.

Our observations being limited to children may not be entirely comparable to other investigations including adults. It may also be possible that the reaction to streptococcal infection in adults, like that to rheumatic fever, may be different from that in children.

\section{SUM MARY}

1. There is presented a correlation of the clinical course with the bacteriological and immunological observations in 80 rheumatic subjects observed over a period of twelve to eighteen months.

2. The antistreptolysin titre for rheumatic subjects during apparent health gave a basal average of 135 units with a range of 25 to 715 units.

3. There was no significant difference in the range of antistreptolysin titre observed for subjects during apparent health and during respiratory and rheumatic infection.

4. A comparison of the antistreptolysin titres of inactive rheumatic subjects during respiratory and "streptococcal" respiratory infections showed a higher average titre and greater rise in titre for subjects experiencing respiratory infection unassociated with hemolytic streptococci in the pharyngeal flora.

5. Two-thirds of the subjects experiencing rheumatic activity unassociated with respiratory infections did not exhibit a rise in antistreptolysin titre.

6. The antistreptolysin titre of active rheumatic subjects experiencing respiratory and " streptococcal " respiratory infections was similar to that observed for inactive rheumtaic subjects experiencing these infections.

7. Following respiratory infections the antistreptolysin curve was characterized by a step-like elevation within one to three weeks of the onset, rising to a peak and falling by lysis within one or two months or remaining plateau-like at intermediate levels for longer periods, showing successive peaks following repeated respiratory infections.

8. The rise of the level of antistreptolysin in the serum following respiratory infections seemed directly related to the extent of the local and constitutional symptoms irrespective of the presence of hemolytic streptococci in the pharyngeal flora.

9. The antistreptolysin curves observed in subjects who developed respiratory infection simultaneously with, or during, rheumatic activity were similar to those described in subjects experiencing respiratory infections alone and bore no relation to the clinical course of rheumatic activity.

\section{CONCLUSION}

1. These observations do not support the assumption that a rise in the antistreptolysin titre of the serum is conclusive evidence of streptococcal respiratory infection.

2. A rise in the antistreptolysin titre is not a necessary accompaniment of rheumatic fever in children.

\section{APPENDIX}

Todd stated in his original article (2) that a serum of known antistreptolysin titre was necessary as a control of the antigen-antibody reaction upon which this test is based. Hodge and Swift (7) proved experimentally that the minimum hemolytic dose is not a reliable unit for standardizing the streptolysin which is used as antigen. They demonstrated that under different conditions of age, oxidation, reduction and temperature a single streptolysin filtrate would show wide variations 
in the minimum hemolytic dose, yet in all instances, practically the same quantity of serum was required to effect complete inhibition of hemolysis. It was evident that under these conditions there were components in the streptolysin that would combine with antistreptolysin although they were not, themselves, hemolytic (partially oxidized streptolysin?). For this reason Hodge and Swift recommended that the combining power, which is stable, and not the hemolytic activity, which is variable, be used as the criterion in the standardization of streptolysin. This would necessitate the adoption of a standard serum of known antistreptolysin content against which all new lots of streptolysin could be titrated, and their combining doses determined. Todd has already made this practicable by his preparation of horse serum globulin, of high antistreptolysin titre, which is remarkably stable.

It appears that the factors involved in lysin-antilysin reactions are analogous to those concerned in toxinantitoxin neutralization. In the latter instance no accurate standardization was possible until the neutralizing dose was replaced by the combining dose, and a standard serum of known antitoxin content was adopted.

\section{Preparation of Reagents}

\section{Rabbit red blood cells:}

On the afternoon before the tests were to be made, blood was withdrawn from the rabbit's heart, placed in a sterile tube, defibrinated by stirring with wooden applicators and stored in refrigerator over night. Next morning the cells were washed six times with sterile 0.9 per cent sodium chloride solution. In the final washing they were centrifugalized 15 minutes at 1500 R.P.M.; they were then diluted to make a 5 per cent suspension.

\section{Streptolysin filtrate:}

The broth in which the streptococci were cultivated was prepared by a modification of the method described by Todd and Hewitt (8). Fresh, finely minced beef hearts, trimmed free of fat, were used instead of horse flesh; these were received from the abattoir the same day the animals were killed. Two liters of distilled water were added to each kilo of meat in an enamelware kettle, the mixture was well stirred and placed in refrigerator over night. Fat particles, if present, were skimmed from the surface, and the mixture was heated slowly over a free flame until the temperature reached $80^{\circ} \mathrm{C}$., where it was kept for 30 minutes. The contents of the kettle were stirred constantly during the heating process.

The fluid was then strained through coarse wire gauze, filtered through Prat-Dumas number 45 filter paper and collected in a tall glass cylinder. Part of the residual fluid was removed from the meat by gentle pressure. The temperature of the infusion after filtration was about $50^{\circ} \mathrm{C}$. The following ingredients were added: proteosepeptone, "Difco," 20 grams per liter, sprinkled on surface and allowed to dissolve; dextrose, sodium chloride, and sodium bicarbonate, 2 grams of each per liter; dibasic sodium phosphate $\left(12 \mathrm{H}_{2} \mathrm{O}\right), 1$ gram per liter. When these were dissolved, reaction was adjusted to $\mathrm{pH} 8.0$ with 10 per cent sodium hydroxide solution. Medium was well stirred and placed in refrigerator over night.

A heavy amorphous sediment had formed, leaving a fairly clear supernatant fluid which was removed with a siphon and filtered through Whatman number 12 filter paper. Medium was sterilized by filtration through a battery of six Chamberland $F$ bougies, using a water vacuum pump. The finished product was collected, in $1500 \mathrm{cc}$. lots, in two-liter flasks closed with two-hole rubber stoppers provided with two right angle glass tubes. The tube to which the suction was applied was filled with non-absorbent cotton to prevent air contamination; the other was covered with a small test tube when not in use. When filtration was completed, flasks were placed in incubator for two days as a sterility test.

Each $1500 \mathrm{cc}$. lot was inoculated with $2.5 \mathrm{cc}$. of an 8-hour culture of hemolytic streptococcus 2 in 0.3 per cent dextrose hormone broth. The inoculation was made through the open tube in the stopper of the flask while gentle suction was being applied to the other tube. After 16 hours incubation flasks were placed in ice water to check growth and culture was filtered through six Chamberland $\mathrm{F}$ bougies. The first $300 \mathrm{cc}$. of filtrate were discarded because of the low streptolysin content (8); the remainder was collected in $1500 \mathrm{cc}$. lots by the same procedure that was used with the broth filtrate. The open tube in the stopper was sealed off in a Bunsen flame, the other tube was connected with a water pump, and strong suction was applied for $21 / 2$ hours, the flasks being immersed in a water bath at $30^{\circ} \mathrm{C}$. Bubbling was vigorous at first, but gradually diminished and finally ceased. Vacuum was released, 1.5 gram of sodium hydrosulphite (Baker) and $15 \mathrm{cc}$. of N/1 sodium hydroxide were added to each $1500 \mathrm{cc}$., and suction was again applied for 30 minutes. The reduced filtrate was then distributed in $50 \mathrm{cc}$. Florence flasks, sealed with a 4 $\mathrm{cm}$. layer of sterile vaseline containing 12 per cent paraffin, and stored in refrigerator.

After three weeks the reaction of this filtrate was $\mathrm{pH} 7.3$; the minimum hemolytic dose was $0.05 \mathrm{cc}$. (kept at $0^{\circ} \mathrm{C}$., this showed no change in ten months).

\section{Antistreptolysin serum:}

Patient's blood was withdrawn with a dry sterile needle and syringe, placed in a sterile tube and allowed to clot. Tube was centrifugalized, serum was removed with a pipette and transferred to another tube which was then closed with a sterile paraffined cork and stored in refrigerator. Before testing the serum the tubes were placed in a water bath at $55^{\circ} \mathrm{C}$. for 15 minutes to inactivate any natural hemolysin that might be present. Repeated determinations made with the same serum

2 We used strain WPRL which we obtained through the kindness of Dr. Hodge of the Rockefeller Institute. This stock culture was kept in blood broth. 
showed that the antistreptolysin titre remained practically constant for at least eight months.

\section{Techrique of test:}

If the combining dose of the streptolysin filtrate was less than $0.2 \mathrm{cc}$., the filtrate was diluted with 0.9 per cent sodium chloride so that 2.5 units of streptolysin were contained in $0.5 \mathrm{cc}$. of the dilution.

Preliminary test: The serum to be tested was inactivated for 15 minutes at $55^{\circ} \mathrm{C}$. Four dilutions were made with 0.9 per cent sodium chloride, 1-10, 1-50, 1-100, 1-500. One cc. of each dilution was placed in serologic tubes, $0.5 \mathrm{cc}$. of streptolysin dilution was added to each, tubes were well shaken and placed in water bath at $37^{\circ}$ C. for 15 minutes. Each tube then received $0.5 \mathrm{cc}$. of 5 per cent suspension of rabbit red blood cells and was placed immediately in a mechanical mixer where the reagents were thoroughly blended for $\mathbf{4 5}$ seconds. Tubes were replaced in water bath, shaken well after $15 \mathrm{~min}$ utes and removed at the end of one hour. Readings were made after contents of tubes had been allowed to settle for about 30 minutes. The highest of the four dilutions that caused complete inhibition of hemolysis was used in the final test.

Final test: The dilution selected was distributed in 10 serologic tubes, beginning with $1.0 \mathrm{cc}$. and diminishing by tenths to $0.1 \mathrm{cc}$. The volumes in the last nine tubes were made up to $1.0 \mathrm{cc}$. with 0.9 per cent sodium chloride. The rest of the procedure was the same as that described for the preliminary test. Tubes were placed in refrigerator, and final readings were made the next morning. The smallest quantity of serum that completely neutralized 2.5 units of streptolysin, thus causing total inhibition of hemolysis, was recorded as the antistreptolysin titre of the serum.

Controls: Each day's tests carried the following controls: One unit of red cells and one unit of streptolysin; no serum. One unit of red cells and 2.5 units of streptolysin; no serum. Preparation of horse serum globulin of known antistreptolysin titre; this was sent to us from the Belmont Laboratories, London, England, through the kindness of Dr. Todd. One or more patients' serums of known antistreptolysin titre.

\section{BIBLIOGRAPHY}

1. Wilson, M. G., Ingerman, E., DuBois, R. O., and Spock, B., The relation of upper respiratory infections to rheumatic fever in children. I. The significance of hemolytic streptococci in the flora of the throat during respiratory infection. J. Clin. Invest., 1935, 14, 325.

2. Todd, E. W., Antigenic streptococcal hemolysin. J. Exper. Med., 1932, 55, 267.

3. Todd, E. W., Antihemolysin titres in hemolytic streptococcal infections and their significance in rheumatic fever. Brit. J. Exper. Path., 1932, 13, 248.

4. Coburn, A. F., and Pauli, R. H., Studies on the relationship of streptococcus hemolyticus to the rheumatic process. I. Observations on the ecology of hemolytic streptococcus in relation to the epidemiology of rheumatic fever. J. Exper. Med., 1932, 56, 609.

5. Myers, W. K., and Keefer, C. S., Antistreptolysin content of blood serum in rheumatic fever and rheumatoid arthritis. J. Clin. Invest., 1934, 13, 155.

6. Wilson, M. G., Wheeler, G. W., and Leask, M. M., Antistreptolysin content of blood serum of children. Its significance in rheumatic fever. Proc. Soc. Exper. Biol. and Med., 1934, 31, 1001.

7. Hodge, B. E., and Swift, H. F., Varying hemolytic and constant combining capacity of streptolysins; influence on testing for antistreptolysins. J. Exper. Med., 1933, 58, 277.

8. Todd, E. W., and Hewitt, L. F., A new culture medium for the production of antigenic streptococcal haemolysin. J. Path. and Bact., 1932, 35, 973. 